\title{
Validation of cone-beam computed tomography as a predictor of osteoporosis using the Klemetti classification
}

\section{Maria Beatriz Carrazzone Cal ALONSO(a)}

Taruska Ventorini VASCONCELOS(a) Luciana Jácome LOPES(a)

Plauto Christopher Aranha WATANABE ${ }^{(b)}$

Deborah Queiroz FREITAS(c)

(a) Universidade de Campinas - UNICAMP, Department of Oral Diagnosis, Oral Radiology Area, Piracicaba Dental School, Piracicaba, SP, Brazil.

(b) Universidade de São Paulo - USP, Ribeirão Preto School of Dentistry, Department of Stomatology, Public Health and Forensic Dentistry, Ribeirão Preto, SP, Brazil.

(c) Universidade de Campinas - UNICAMP, Piracicaba Dental School, Department of Oral Diagnosis, Piracicaba, SP, Brazil.

Declaration of Interests: The authors certify that they have no commercial or associative interest that represents a conflict of interest in connection with the manuscript.

Corresponding Author: Luciana Jácome Lopes E-mail: lu_jacome@hotmail.com

DOI: 10.1590/1807-3107BOR-2016.vol30.0073

Submitted: Sep 16, 2015

Accepted for publication: Mar 07, 2016

Last revision: Apr 30, 2016
Abstract: This study aimed at evaluating the validity of cone-beam computed tomography (CBCT) for assessing mandibular bone quality using the Klemetti classification. The morphology of the endosteal mandibular cortex of 30 (60 hemi-mandibles) postmenopausal women between the ages of 45 and 80 years was evaluated based on the Klemetti classification in panoramic radiographs used as reference images. Afterwards, panoramic reconstruction and cross-sectional slices of CBCT examinations of these patients were analyzed and categorized according to the same classification. All the images were assessed by two oral radiologists. The McNemar-Bowker test compared the agreement between the CBCT images and the reference images. No differences were found between the diagnostic results based on panoramic radiography and panoramic reconstruction. However, the mean scores for the cross-sectional evaluation were higher, and the results, statistically different from the others. Based on the disagreement found between the panoramic radiographs and the CBCT cross-sectional slices, the Klemetti classification is not an adequate means of assessing bone quality with CBCT. On the other hand, the higher values found for the cross-sectional slices could be associated with better visibility on the CBCT images.

Keywords: Cone-Beam Computed Tomography; Osteoporosis; Bone and Bones; Radiography, Panoramic.

\section{Introduction}

Osteoporosis is a major health problem and affects a significant number of people. This skeletal disorder is characterized by bone fragility caused by deterioration of the bone micro-architecture, which imparts strength and bone quality. This condition can increase the risk of and is associated with certain serious complications, resulting in death. ${ }^{1,2}$ Screening for osteoporosis is currently recommended for all women aged 65 years or older. The complications of this disease may be prevented by early detection. ${ }^{1}$ Diagnosis is currently based mainly on the measurement of bone mineral density (BMD) using dual-energy $\mathrm{X}$-ray absorptiometry (DXA), but bone densitometry is expensive and is of limited availability for routine use in population screening., 
Because osteoporosis is a systemic skeletal disease, the bone density and structure of the jaw are affected. The BMD of the mandible is correlated with that of the lumbar spine and femoral neck in osteoporosis. ${ }^{5}$ Several studies have demonstrated that individuals with osteoporosis have altered morphology of the mandible, and there is evidence that intraoral and panoramic radiographic findings could be indicators of osteoporosis. ${ }^{1,6,7,8}$ Panoramic radiography (PAN) stands out in this context, because it is frequently conducted in the dental office, is quick and inexpensive, and uses low-dose $X$-radiation. The most commonly studied qualitative measure of mandibular morphology in relation to osteoporosis is the integrity of the inferior border. Erosions of the inferior border are typically scored using the mandibular cortical index. ${ }^{7}$ This index (known as the Klemetti index) was established by Klemetti et al. ${ }^{9}$ and developed for PAN in a study in which the authors concluded that individuals with osteoporosis are also more likely to show erosions; thus, individuals could be classified into three distinct groups: $\mathrm{C} 1$ - the margin of the cortex is clear and sharp on both sides; C2 - endosteal surface defects are semilunar; C3 - the cortical layer is extremely porous. The authors suggested that this evaluation is useful for identifying postmenopausal women with undetected low skeletal BMD or osteoporosis. It is expected that the discovery of predictive, low mineral density studies that improve the clinical application of existing methods can contribute to the detection of patients at risk for low bone mass. Thus, dentists could identify and refer asymptomatic patients as the most appropriate patients to receive medical treatment. ${ }^{9}$

For over a century, conventional dental radiographs have been the dominant source of diagnostic information on the maxillofacial complex. ${ }^{10}$ Cone-beam computed tomography (CBCT) is a more recent development, and its clinical application in the field of dentomaxillofacial radiology is gaining importance and becoming widespread. ${ }^{11}$ Although the clinical relevance of dental diagnosis and presurgical assessment of jaw bone density has been clearly demonstrated, the available research on CBCT-based bone quality is scant. ${ }^{2}$ A new tool, the computed tomography index, was proposed to assess the BMD on CBCT images; ${ }^{5}$ with it, CBCT images can be used to evaluate women with osteoporosis, thus increasing the possibilities for screening and early detection. A recent study evaluated the mandibular index on CBCT images and found that the use of this index in CBCT images was valid; ${ }^{12}$ however, the index obtained from CBCT images did not compare well with that derived from PAN, the imaging modality for which the classification was originally devised. Moreover, the CBCT analysis was conducted in templates, i.e., static slices. These aspects could have influenced the results. ${ }^{12}$

A systematic review evaluated the accuracy (relative to DXA) of the different panoramic morphometric indices, and, based on the findings, suggested future studies to examine ways of controlling the limitation of the magnification/distortion of PAN in order to attain more reliable and precise conclusions. ${ }^{13}$ Seeking to address this limitation, the present study aims at evaluating the validity of CBCT in assessing mandibular bone quality using the Klemetti classification of inferior mandible morphology for the diagnosis of mandibular bone quality. To this end, the classification obtained from CBCT images was compared with that derived from panoramic images.

\section{Methodology}

\section{Patients and imaging procedure}

Approval for the study was obtained from the local Ethical Research Committee, under protocol no. $138 / 2009$. The patient sample was selected from women referred to the Radiological Service of the Piracicaba Dental School, Universidade de Campinas - Unicamp. Inclusion of the patients in the study required their having undergone CBCT with a field of view in which the mandibular cortex was clearly visible; these images were taken for different treatment purposes not related to this study. Patients also had to have undergone PAN within the previous 6 months. Moreover, they had to be over 45 years old, healthy and postmenopausal. Patients presenting with metabolic bone diseases, such as hyper- or hypoparathyroidism, diabetes, osteomalacia or renal disease were excluded from this study. A total of 
30 women met the inclusion criteria, ranging in age from 45 to 80 years (median 62 years).

Digital PAN were acquired by Orthopantomograph OP100 D, (Instrumentarium Corp., Imaging Division, Tuusula, Finland). The CBCT examinations were performed with an i-CAT CBCT unit (Imaging Sciences International, Inc., Hatfield, USA), using the exposure parameters recommended by the manufacturer: $120 \mathrm{kVp}, 3-8 \mathrm{~mA}$; voxel size $0.25 \mathrm{~mm}$; and all the volumes were exported in a DICOM pattern so that the evaluation could be performed.

\section{Image analysis}

Two previously calibrated oral and maxillofacial radiologists with experience using the Klemetti index evaluated the images. The type of inferior mandibular cortex on each side of the patient's posterior mandible for each imaging modality (PAN, panoramic reconstruction (PR) and cross-sectional slices (CS) on CBCT) was classified subjectively according to Klemetti et al.: ${ }^{9}$

$\mathrm{C} 1$ - the endosteal margin of the cortex appears even and sharp;

$\mathrm{C} 2$ - the endosteal margin shows semilunar defects or 1 to 3 layers of cortical endosteal residues; and C3 - the cortical layer has numerous (>3) endosteal residues and is clearly porous.

The assessment of each imaging modality was done separately, in random sequence. First, PAN evaluations were conducted using ImageJ software (National Institutes of Health, Bethesda, MD). The examiners then analyzed the PR images using CS 3D Imaging Software 3.4.3 (Carestream Health Inc., Rochester, USA). To this end, a single examiner selected the axial section corresponding to the opening of the mental foramen, and drew a line in the center of the mandible to assure standardized PR images. The thickness of the PR view used was 2.2 millimeters.

Finally, the CS images were evaluated with the same software used for the PR images. A dynamic evaluation was performed on the CS images with a slice thickness of $200 \mu \mathrm{m}$. The appropriate assessment of the inferior mandibular cortex was performed on each side, according to the CS, involving the opening of the mental foramen up to the third molar region. The observer was allowed to use the "zoom" tool and change the brightness and contrast of the images; however, the enhancement filters were not applied.

All of the evaluations were conducted on a 24.1-inch LCD monitor (MDRC-2124, Barco N.V., Courtray, Belgium) with a resolution of $1920 \times 1200$ pixels in two steps: first, each evaluator classified the images independently; then their results were compared and a consensus was reached. When consensus could not be reached between the two examiners, a third oral and maxillofacial radiologist assisted in making the decision.

After six months, $30 \%$ of the sample was reevaluated to assess the intraobserver agreement.

\section{Statistical methods}

The data were tabulated in the SPSS version 17.0 (SPSS Inc., Chicago, USA) for analysis. Contingency cross-tables that included the scores for both images tested (PR and CS of CBCT) and the reference image (PAN) were created. The McNemar-Bowker test compared agreement between the images and the reference image. The intraobserver agreement was tested by the weighted kappa test. The significance level was set at $5 \%$. The null hypothesis was that there was no disagreement between the images tested and the reference image.

\section{Results}

The $\mathrm{C} 2$ and $\mathrm{C} 3$ scores were the most prevalent in the sample $(86.7 \%)$ when the mandibular cortex was evaluated in the reference images (PAN).

The responses for the PR and CS images were compared with those from the reference images in Tables 1 and 2, respectively. The PR images were in agreement with the PAN images in $65 \%$ of the cases; the agreement between CS and PAN was $56.7 \%$. The McNemar-Bowker test indicated that the CS images were not in agreement with the reference images $(p=0.005)$, unlike the PR images $(p=0.246)$.

The main disagreements between CS and PAN were when PAN indicated $\mathrm{C} 1$, and CS indicated $\mathrm{C} 2$, and when PAN indicated C2, and CS indicated C3. In other words, in several cases, the CS image overestimated the index classification; $96.6 \%$ of the scores were C2 and C3 in the CS images, and 86.7\% with PAN. 
Table 1. Contingency table presenting the responses obtained with PR (panoramic reconstruction) using PAN (panoramic radiography) for the mandibular index (bold letters indicate agreement among the images).

\begin{tabular}{|c|c|c|c|c|}
\hline \multirow{2}{*}{ PR } & \multicolumn{3}{|c|}{ PAN } & \multirow{2}{*}{ Total, n (\%) } \\
\hline & $\mathrm{Cl}, \mathrm{n}(\%)$ & C2, n (\%) & C3, n (\%) & \\
\hline $\mathrm{Cl}$ & $5(8.3)$ & 10 (16.7) & $1(1.7)$ & $16(26.7)$ \\
\hline $\mathrm{C} 2$ & $3(5)$ & $25(41.7)$ & $4(6.6)$ & 32 (53.3) \\
\hline $\mathrm{C} 3$ & $0(0)$ & $3(5)$ & $9(15)$ & $12(20)$ \\
\hline Total & 8 (13.3) & 38 (63.4) & $14(23.3)$ & $60(100)$ \\
\hline
\end{tabular}

$\mathrm{p}=0.246$ (not considered statistically significant according to the McNemar-Bowker test). Bold letters indicate agreement among the images

Table 2. Contingency table presenting the responses obtained with CS (cross-sectional slices) using PAN (panoramic radiography) for the mandibular index (bold letters indicate agreement among the images).

\begin{tabular}{|c|c|c|c|c|}
\hline \multirow{2}{*}{ CS } & \multicolumn{3}{|c|}{ PAN } & \multirow{2}{*}{ Total, n (\%) } \\
\hline & $\mathrm{Cl}, \mathrm{n}(\%)$ & C2, n (\%) & C3, n (\%) & \\
\hline $\mathrm{Cl}$ & 1 (1.7) & $1(1.7)$ & $0(0)$ & $2(3.4)$ \\
\hline $\mathrm{C} 2$ & 7 (1 1.6) & $22(36.7)$ & $3(5)$ & 32 (53.3) \\
\hline C3 & $0(0)$ & $15(25)$ & $11(18.3)$ & $26(43.3)$ \\
\hline Total & 8 (13.3) & $38(63.4)$ & $14(23.3)$ & $60(100)$ \\
\hline
\end{tabular}

$p=0.005$ (not considered statistically significant according to the McNemar-Bowker test). Bold letters indicate agreement among the images.

The intraobserver agreement for the weighted kappa test was substantial for CS (0.76), and almost perfect for PAN and PR (0.95 and 0.91, respectively), according to Landis and Koch. ${ }^{14}$

Figures 1 and 2 show examples of the Klemetti index evaluated in each imaging modality.

\section{Discussion}

Early detection of osteopenia and osteoporosis is important to decrease the risk of fracture and prevent pain and disability. ${ }^{5,15}$ According to the World Health Organization (WHO), one-third of women over the age 65 years are affected by osteoporosis. ${ }^{16}$ Because life expectancy is increasing throughout the world, the number of elderly individuals is also increasing worldwide, thus making osteoporosis a global public health problem.

DXA is a well-established method for measuring BMD in the femoral neck and spine. ${ }^{17}$ However, because of the cost and lack of availability in some regions, DXA measurements are considered less suitable for large-scale screening. ${ }^{18}$ Several studies have proposed assessment methods that allow early detection of systemic loss of $\mathrm{BMD}$, and suggested that evaluation of the quality of the jaw bones using dental radiographs could be an important screening method for detecting osteopenia or osteoporosis. . $^{8,919,20,21,22,23}$

The cortical area of the mandible helps assess osteoporosis on PAN images. Radiomorphometric indexes were developed for PAN to assess skeletal BMD or osteoporotic status through qualitative and quantitative measurements, similar to the Klemetti index, which is based on a qualitative analysis of the cortical area of the mandible. ${ }^{9}$ This index has already proven to be a useful tool in screening for osteopenia or osteoporosis, compared with the BMD of the lumbar spine, as measured by DXA according to several studies.1,15,24,25,26 The general purpose and the clinical significance of the Klemetti index (an ordinal classification for defining osteoporosis) is based on the evaluation of the methods available to users of panoramic $x$-ray images for estimating changes in cortical bone minerals of the mandible. According to the index, the general mineral density may be 

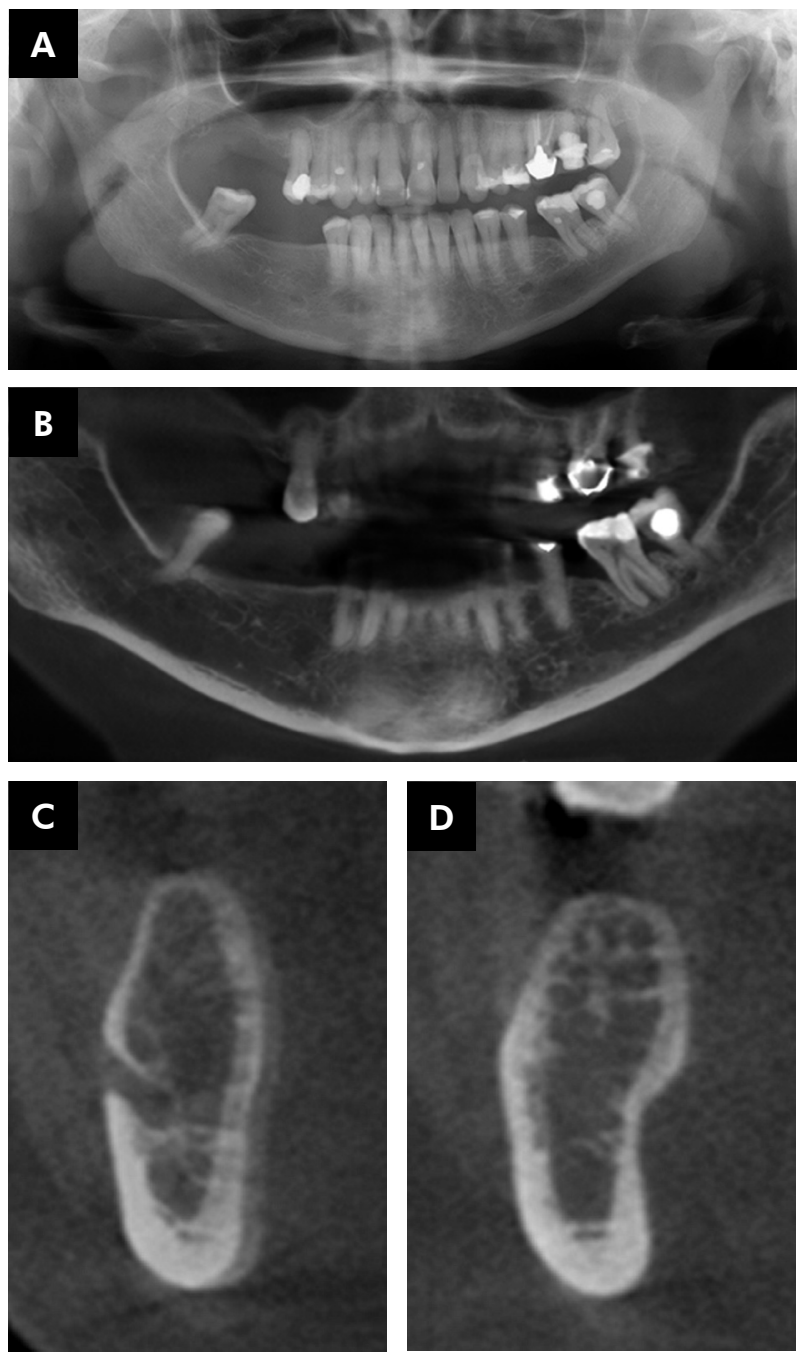

Figure 1. Examples of agreement classification obtained in the imaging modalities studied in the $C 2$ category (presence of semilunar defects in the endosteal margin): (A) PAN; (B) - PR; (C) - CS (right); (D) - CS (left).

predicted when skeletal changes occur in the bone structures on panoramic X-ray images. The mineral loss in the mandibular cortex seems to depend on the speed of mineral loss in the skeleton and on age. ${ }^{9}$ The rate of residual ridge reduction may also be predicted from the initial mandibular bone mineral cortex. In the region distal to the mental foramen, the buccal cortex has been reported to correlate better with skeleton mineral density values than the lingual portion. ${ }^{27}$ On panoramic X-ray images, the inferior cortex is a reflection of an area that represents neither of these (buccal cortex nor lingual portion). However, the inferior cortex of the mandible seen on
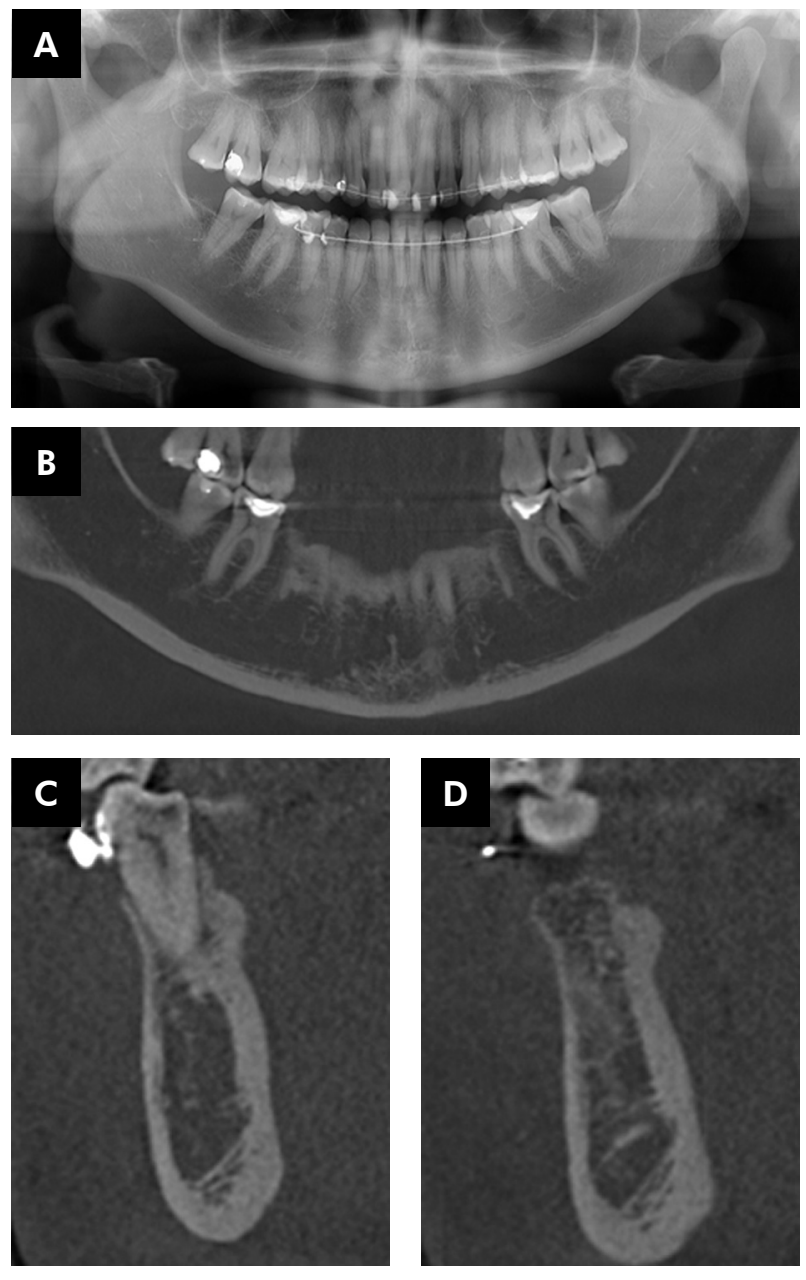

Figure 2. Example of discordance between the images using the Klemetti index: in PAN (A) and PR (B), the case was classified as $C 2$; in CS ((C) right; (D) left), the classification was C3.

these images is the most suitable bone structure for this study because this is clearly visible. ${ }^{9}$

Considering that the Klemetti index is reliable as a predictor of osteoporosis on PAN, and that the clinical application of CBCT in the field of dentomaxillofacial radiology is gaining importance and becoming widespread, ${ }^{11}$ the present study compared the validity of the Klemetti index on CBCT. Even though the density measurements (in Hounsfield units) are not valid for $\mathrm{CBCT}$, the $\mathrm{CBCT}$ per se shows a clear image of highly contrasted structures, and is extremely useful for evaluating the bone. ${ }^{27} \mathrm{CBCT}$ data are amenable to reformation in a volume, rather than a slice, providing three-dimensional information, and the resulting images are useful in assessing specific 
morphological features such as alveolar bone height and width. ${ }^{11}$

Hua et al. ${ }^{2}$ proposed texture analysis based on the fact that bone quality may be expressed by the composition of its micro-architecture. They demonstrated that fractal analysis and bone area measurements have the potential to evaluate bone quality on CBCT images, whereas density measurements do not seem to be valid. Koh and $\mathrm{Kim}^{5}$ published the first study using CBCT in the evaluation of BMD in postmenopausal women with osteoporosis. They adapted the qualitative and quantitative radiomorphometric index used on PAN images to the CBCT images and compared the results with the densitometry of normal and osteoporotic women. They suggested that CBCT images can be used to assess osteoporosis in women. The results of the present study showed differences between the diagnoses using the Klemetti index on CS compared with PAN, indicating that this index may not be valid for CBCT assessments. However, this does not mean that the CBCT is not useful for evaluating the mandible cortical. On the contrary, our results also indicated that changes in the morphology of the mandibular cortex can be detected by CBCT; the mean score for CS was the highest in this evaluation. This shows that the failure of this type of CBCT assessment was not based on the poor detection of changes.

In the present study, panoramic images were used and CBCT images were evaluated by a good software program, allowing the examiners to analyze the entire volume and make a dynamic evaluation. The present study took into account the inherent differences in the two imaging modalities. Whereas CBCT reproduces structures (even considering the presence of some technical limitations) in their original size, the final image of the PAN is influenced by magnification and distortion, due to the characteristics of the image formation. Thus, it was not expected that the Klemetti classification would be transferable to CBCT; however, a study comparing the assessment of the Klemetti index on PR and CS from CBCT examinations in an osteoporosis risk group drew our attention, because we did not find significant differences between the examinations. ${ }^{12}$ Although, the images were evaluated on a template, which did not allow the examiners to analyze multiple CS, this could limit the diagnostic potential; moreover, the only panoramic image used was PR, which has different physics-related and imaging principles from the classic panoramic images used for the Klemetti index. This limitation was indicated by the authors, and led us to develop this investigation.

The results obtained in this study could be influenced by two distinct factors; we believe the capacity of CS images to show changes of this type if the bone micro-architecture is clearly visible without overlapping structures. However, it is not possible to ignore the occurrence of a large amount of false-positive diagnoses. These two factors do not negate the usefulness of these kinds of image for analyzing the mandibular cortex to predict osteoporosis, but do indicate the need for more studies designed to create an index appropriate for CBCT use.

The success of an imaging classification method is influenced by the reproducibility capacity, whose accuracy should not be affected by factors related to image evaluation criteria, such as evaluator doubt or change of opinion. Although the assessment of bone quality is a subjective procedure, the intraobserver agreement proved that the evaluation was performed by trained professionals who provided precise results. In addition, this bone structure assessment method may be considered a reliable tool.

The mandibular cortical index in osteoporotic women receiving oral bisphosphonates was quantitatively evaluated on CBCT images. ${ }^{29}$ A significant increase in the cortical area was observed, as expected, seeing that the assessment was carried out on CS instead of the PR used in most studies. This shows the importance of CS and their value to the dentistry community, as well as the appropriate use of all the features of this imaging method.

\section{Conclusion}

The Klemetti index should not be used to assess osteoporosis on CBCT cross-sectional slices; on the other hand, this imaging modality has great potential for performing osteoporosis analysis, because the inferior mandible cortex is given visibility in its all extension by dynamic evaluation. 


\section{References}

1. Erdogan O, Incki KK, Benlidayi ME, Seydaoglu G, Kelekci S. Dental and radiographic findings as predictors of osteoporosis in postmenopausal women. Geriatr Gerontol Int. 2009;9(2):155-64. doi:10.1111/j.1447-0594.2009.00518.x

2. Hua Y, Nackaerts O, Duyck J, Maes F, Jacobs R. Bone quality assessment based on cone beam computed tomography imaging. Clin Oral Implants Res. 2009;20(8):767-71. doi:10.1111/j.1600-0501.2008.01677.x

3. Reginster JY, Burlet N. Osteoporosis: a still increasing prevalence. Bone. 2006;38(2 Suppl 1):S4-9. doi:10.1016/j.bone.2005.11.024

4. Nakamoto T, Taguchi A, Ohtsuka M, Suei Y, Fujita M, Tanimoto K, et al. Dental panoramic radiograph as a tool to detect postmenopausal women with low bone mineral density: untrained general dental practitioners' diagnostic performance. Osteoporos Int. 2003;14(8):659-64. doi:10.1007/s00198-003-1419-y

5. Koh KJ, Kim KA. Utility of the computed tomography indices on cone beam computed tomography images in the diagnosis of osteoporosis in women. Imaging Sci Dent. 2011;41(3):101-6. doi:10.5624/isd.2011.41.3.101

6. Dagistan S, Bilge OM. Comparison of antegonial index, mental index, panoramic mandibular index and mandibular cortical index values in the panoramic radiographs of normal males and male patients with osteoporosis. Dentomaxillofac Radiol. 2010;39(5):290-4. doi:10.1259/dmfr/46589325

7. White SC. Oral radiographic predictors of osteoporosis. Dentomaxillofac Radiol. 2002;31(2):84-92. doi:10.1038/sj.dmfr.4600674

8. Horner K, Devlin H, Harvey L. Detecting patients with low skeletal bone mass. J Dent. 2002;30(4):171-5. doi:10.1016/ S0300-5712(02)00010-6

9. Klemetti E, Kolmakov S, Kröger H. Pantomography in assessment of the osteoporosis risk group. Scand J Dent Res. 1994;102(1):68-72. doi: 10.1111/j.1600-0722.1994.tb01156.x

10. Jacobs R. Dental cone beam CT and its justified use in oral health care. JBR-BTR. 2011;94(5):254-65. doi: $10.5334 /$ jbr-btr.662

11. Scarfe WC, Farman AG, Sukovic P. Clinical applications of cone-beam computed tomography in dental practice. J Can Dent Assoc. 2006;72(1):75-80.

12. Gomes CC, Barbosa GLR, Bello RP, Bóscolo FN, Almeida $\mathrm{SM}$. A comparison of the mandibular index on panoramic and cross-sectional images from CBCT exams from osteoporosis risk group. Osteoporos Int. 2014;25(7):1885-90. doi:10.1007/s00198-014-2696-3

13. Calciolari E, Donos N, Park JC, Petrie A, Mardas N. Panoramic measures for oral bone mass in detecting osteoporosis: a systematic review and meta-analysis. J Dent Res. 2015;94(3 Suppl):17S-27S . doi:10.1177/0022034514554949
14. Landis JR, Koch GG. The measurement of observer agreement for categorical data. Biometrics. 1977;33(1):159-74. doi:10.2307/2529310

15. Halling A, Persson GR, Berglund J, Johansson O, Renvert S. Comparison between the Klemetti index and heel DXA BMD measurements in the diagnosis of reduced skeletal bone mineral density in the elderly. Osteoporos Int. 2005;16(8):999-1003. doi:10.1007/s00198-004-1796-x

16. World Health Organization Expert Commitee on Biological Standardization. Assessment of fracture risk and its applications to screening for post menopausal osteoporosis. Geneva: WHO; 1994. 129 p. (Technical Report Series).

17. Horner K, Karayianni K, Mitsea A, Berkas L, Mastoris $\mathrm{M}$, Jacobs $\mathrm{R}$, et al. The mandibular cortex on radiographs as a tool for osteoporosis risk assessment: the OSTEODENT Project. J Clin Densitom. 2007;10(2):138-46. doi:10.1016/j.jocd.2007.02.004

18. Nackaerts O, Gijbels F, Sanna AM, Jacobs R. Is there a relation between local bone quality as assessed on panoramic radiographs and alveolar bone level? Clin Oral Investig. 2008;12(1):31-5. doi:10.1007/s00784-007-0139-7

19. Taguchi A, Suei Y, Ohtsuka M, Otani K, Tanimoto $\mathrm{K}$, Ohtaki M. Usefulness of panoramic radiography in the diagnosis of postmenopausal osteoporosis in women. Width and morphology of inferior cortex of the mandible. Dentomaxillofac Radiol. 1996;25(5):263-7. doi:10.1259/dmfr.25.5.9161180

20. Horner K, Devlin H. The relationship between mandibular bone mineral density and panoramic radiographic measurements. J Dent. 1998;26(4):337-43. doi:10.1016/S0300-5712(97)00020-1

21. Ledgerton D, Horner K, Devlin H, Worthington H. Radiomorphometric indices of the mandible in a British female population. Dentomaxillofac Radiol. 1999;28(3):173-81. doi:10.1038/sj.dmfr.4600435

22. Güngör K, Akarslan Z, Akdevelioglu M, Erten H, Semiz M. The precision of the panoramic mandibular index. Dentomaxillofac Radiol. 2006;35(6):442-6. doi:10.1259/dmfr/25346328

23. Gulsahi A, Yüzügüllü B, Imirzalioglu P, Genç Y. Assessment of panoramic radiomorphometric indices in Turkish patients of different age groups, gender and dental status. Dentomaxillofac Radiol. 2008;37(5):288-92. doi:10.1259/dmfr/19491030

24. Taguchi A. Triage screening for osteoporosis in dental clinics using panoramic radiographs. Oral Dis. 2010;16(4):316-27. doi:10.1111/j.1601-0825.2009.01615.x

25. Taguchi A, Tsuda M, Ohtsuka M, Kodama I, Sanada M, Nakamoto T, et al. Use of dental panoramic radiographs in identifying younger postmenopausal women with osteoporosis. Osteoporos Int. 2006;17(3):387-94. doi:10.1007/s00198-005-2029-7 
26. Leite AF, Figueiredo PTS, Guia CM, Melo NS, Paula AP.

Correlations between seven panoramic radiomorphometric indices and bone mineral density in postmenopausal women. Oral Surg Oral Med Oral Pathol Oral Radiol Endod. 2010;109(3):449-56. doi:10.1016/j.tripleo.2009.02.028

27. Sukovic P. Cone beam computed tomography in craniofacial imaging. Orthod Craniofac Res. 2003;6(Suppl 1):31-6. doi:10.1034/j.1600-0544.2003.259.x

28. Klemetti E, Kolmakow S. Morphology of the mandibular cortex on panoramic radiographs as an indicator of bone quality. Dentomaxillofac Radiol. 1997;26(1):22-5. doi:10.1038/sj.dmfr.4600203

29. Diniz-Freitas M, Fernández-Montenegro P, Fernández-Feijoo J, Limeres-Posse J, González-Mosquera A, Vázquez-García E, et al. Mandibular cortical indices on cone-beam computed tomography images in osteoporotic women on treatment with oral bisphosphonates. Gerodontology [Internet]. 2014 Apr 4 [cited 201604 07]. Avalilable from: http://onlinelibrary.wiley.com/doi/10.1111/ ger.12121/abstract. doi:10.1111/ger.12121 